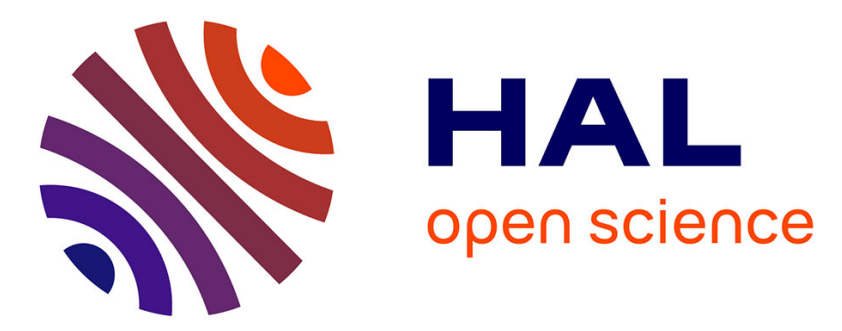

\title{
Transparent and Dynamic Deployment of Lightweight Transport Protocols
}

El-Fadel Bonfoh, Djolo Cédric Tape, Christophe Chassot, Samir Medjiah

\section{To cite this version:}

El-Fadel Bonfoh, Djolo Cédric Tape, Christophe Chassot, Samir Medjiah. Transparent and Dynamic Deployment of Lightweight Transport Protocols. 2019 IEEE Global Communications Conference (GLOBECOM), Dec 2019, Waikoloa, Hawaii, United States. hal-02378283

\section{HAL Id: hal-02378283 \\ https://hal.science/hal-02378283}

Submitted on 27 Nov 2019

HAL is a multi-disciplinary open access archive for the deposit and dissemination of scientific research documents, whether they are published or not. The documents may come from teaching and research institutions in France or abroad, or from public or private research centers.
L'archive ouverte pluridisciplinaire HAL, est destinée au dépôt et à la diffusion de documents scientifiques de niveau recherche, publiés ou non, émanant des établissements d'enseignement et de recherche français ou étrangers, des laboratoires publics ou privés. 


\section{Transparent and Dynamic Deployment of Lightweight Transport Protocols}

\author{
El-Fadel Bonfoh \\ LAAS-CNRS \\ University of Toulouse \\ Toulouse, France \\ efbonfoh@laas.fr
}

\author{
Djolo Cédric Tape \\ LAAS-CNRS \\ University of Toulouse \\ Toulouse, France \\ dctape@laas.fr
}

\author{
Christophe Chassot \\ $L A A S-C N R S$ \\ University of Toulouse \\ Toulouse, France \\ chassot@laas.fr
}

\author{
Samir Medjiah \\ LAAS-CNRS \\ University of Toulouse \\ Toulouse, France \\ medjiah@laas.fr
}

\begin{abstract}
The adoption of new Transport protocols on the Internet remains a critical challenge and their effective deployment is very slow. Till now, despite its known limitations and the plethora of existing alternatives like QUIC or DCTCP, almost $90 \%$ of applications transmissions are based on TCP. From this observation, we assert that redirecting TCP-connection to another Transport protocol may accelerate the deployment and the adoption of any new Transport protocols in the Internet. The selected Transport protocol towards which the redirection is performed may either already exist in the OS or dynamically be deployed on it. Recently introduced in the Linux kernel, eBPF technology provides the abilities to insert at runtime functionalities in the kernel from userspace programs. In this paper, we propose a preliminary design of a eBPF-based framework to perform our approach. Following this design, we implement a prototype that safely (1) perform transparent redirection of TCP connections either to the OS native UDP or to UDP-Lite; the later one is (2) dynamically deployed as eBPF programs. This first prototype, developed on Linux 5.0, has the worth to demonstrate our concept but suffer from performances issues to which we formulate some solutions and open up the associated research questions. Nevertheless, we believe that our approach may lead to innovation at the Internet Transport layer.
\end{abstract}

Keywords - Transport protocols; TCP/UDP; UDP-Lite; eBPF; dynamic deployment; Linux $O S$

\section{INTRODUCTION}

The Internet has known rapid and continuous development of Transport protocols not only to satisfy the functional requirements but also to meet applications QoS and, more recently, QoE requirements. Unfortunately, there is a wide gap between the development and the speed of deployment/adoption of those protocols. The Transport layer is only based on TCP and UDP, and nearly $90 \%$ of Internet traffic is based on TCP [1]. Deployment and adoption of new Transport protocols are slow and difficult.

Indeed, to support any new protocol, OS developers have to add it to the OS kernel; this is so tedious and error-prone to the point that it can only be motivated by high demand from application programmers. At the same time, for the application programmers, modifying an application to support any new protocol is time-consuming

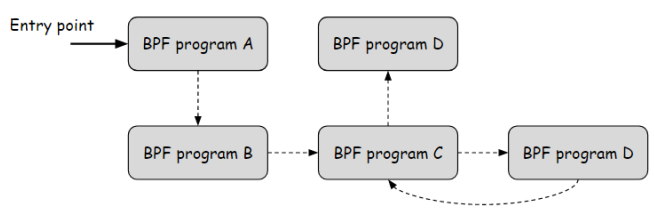

Fig. 1. eBPF programs chain by tail calls [15]

and quite complex [2]; this leads them to definitively use TCP despite its well-known limitations in many modern networks context [3].

As if it was not already complicated enough, besides these two actors, there are middleboxes vendors which come to "violate" the end-to-end principle of the Transport layer; indeed, their devices (NAT, Firewall, etc.) usually block all the Transport protocols that are not supported by mainstream OS developers (Windows, Mac, Linux, etc.). This global context leads to a vicious circle that hampers the deployment and the adoption of any new Transport solutions other than TCP or UDP [4, 5], explaining the lack of innovation within the Internet Transport layer.

Given the huge number of TCP-based applications, we assert that the capability to, transparently for applications and safely for the system, redirect TCP connections to another Transport protocol can definitely accelerate the deployment and encourage the adoption of novel Transport protocols in the Internet. The selected Transport protocol towards which the redirection is performed may either already exist in the OS or dynamically be deployed on it. Indeed, every time a new protocol is released, thanks to dynamic deployment features, the OS developers do not have necessarily to upgrade their systems and the applications programmers can leverage the benefits of the new protocol without modifying their applications thanks to transparent redirection capabilities. To be complete and viable, this approach may integrate a systematic fallback to TCP in case of any failure (technical, middleboxes interferences, etc.). It is important to specify at this level that the prototype presented in this paper does not include this complementary mechanism. 


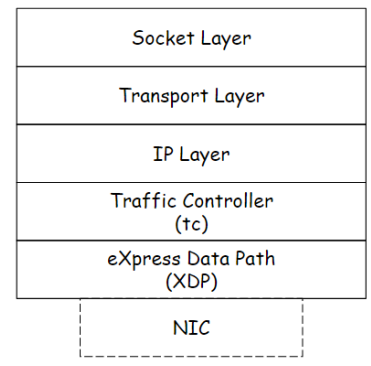

Fig. 2. Traffic Control and XDP

More recently, Linux extended the BPF [6] virtual machine to the know as eBPF. In short, eBPF allow to dynamically add user code in the kernel and so temporary modified its behavior. More about eBPF architecture and functioning is provided in the background section.

To show the feasibility of our approach, we preliminary design and implement a eBPF-based tool on Linux 5.0. This tool, which we call Hooker is a part of Virtual Transport Layer described in [4]. The Hooker framework leverages eBPF [7] features to transparently and safely redirect TCP connections to the OS native UDP or to the dynamically deployed UDP-Lite. The selected protocol could be any other than UDP and UDP-Lite. Through an illustrative data streaming scenario, we test our prototype and successfully perform the transparent redirection of TCP connections and the dynamic deployment of UDPLite as eBPF programs. Despite (1) the potential of our approach to make it easier the deployment of new Transport solutions, and then and to stimulate their adoption, and (2) its subsequent benefits in terms of flexibility, customization, and protocol adaptation, the current prototype face performance issues. In this paper, we formulate some preconizations and open up the related research questions.

The rest of the paper is organized as the following. The next section presents the background to this work and an overview of the related work. Section III presents the details of the preliminary design and implementation of the Hooker framework. In section IV, functional tests and performances analysis of the Hooker are presented. Finally, section V concludes the paper and enumerates the perspectives of this work and related opened research questions.

\section{BACKGROUND AND RELATED WORK}

\section{A. Background}

The Hooker framework developed in this work is based on eBPF. eBPF (extended Berkeley Packet Filter), as suggested by its name, is an extension of BPF in-kernel virtual machine allowing to inject bytecode within Linux kernel at runtime. Its main components are: (1) maps which are key / value stores used to exchange data either between user-space programs and in-kernel eBPF programs, or between eBPF programs running at different points of the kernel, (2) helper functions executed directly inside the kernel and allowing eBPF programs to interact with it, and (3) tail calls used to chain up to $32 \mathrm{eBPF}$ programs as shown in Fig. 1. The latter feature can be used to overcome the size limitation of eBPF program, fixed to 4096 bytes.

$\mathrm{eBPF}$ is used in several contexts like tracing, monitoring, security, and networking. Each eBPF program may be attached to a hook point, a.k.a as kernel event (incoming packet, system calls, socket operations, ...), and each time the event occurs, the eBPF program is executed. The two main networking hook points are eXpress Data Path (XDP) [8] and Traffic Controller (tc) [9] which are placed at different levels of the networking stack (see Fig. 2).

eBPF introduces programmability in the Linux kernel by allowing runtime code injection within the kernel and so, as illustrated in Fig. 3, provides the ability to dynamically add functionalities in the kernel from userspace programs compiled by LLVM/Clang compiler. Thanks to JIT compiler and eBPF Verifier, these functionalities are safely added and efficiently executed. Furthermore, eBPF has the advantage of allowing temporary kernel modification as opposed to kernel patches approach.

To dynamically deploy Transport functions in the OS kernel, we first considered the use of Linux kernel modules; permitting seamlessly integration and utilization of the introduced Transport functions. Unfortunately, we faced the most common issue of kernel module utilization: the whole system crashed at the slightest mistake; in other words, there is no verifier to guarantee the safety of the OS. Furthermore, there is a lack of debug tools and it takes time to check the code and to repair the bug.

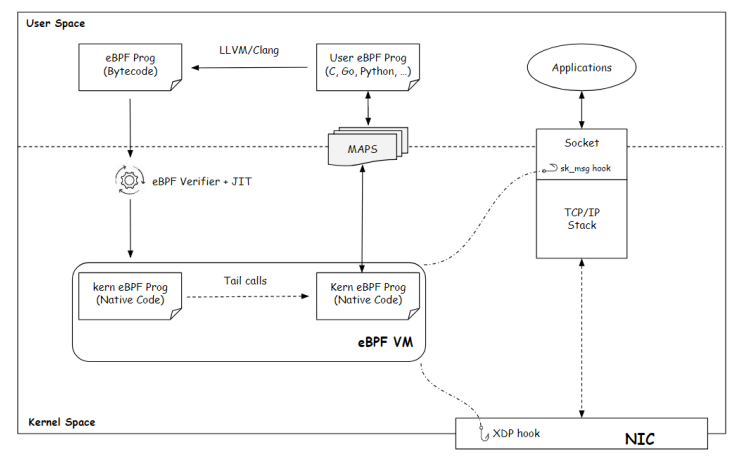

Fig. 3. eBPF Networking Overview

\section{B. Related work}

Various works have been done to tackle the issue of deployment and adoption of Transport protocols. [10,11] propose TCP-SCTP mapping tool for transparent redirection of TCP connection to SCTP in order to gradually deploy SCTP. In [10], the mapping tool acts like a proxy which merges individual TCP connections into SCTP association whereas in [11] the mapping tool is designed to be directly integrated into the OS. However, such approaches address only SCTP deployment and adoption 
issues; they do not provide a comprehensive way for mapping to other protocols than SCTP.

More recent works $[12,13,2]$ propose to rethink the entire Transport layer architecture in order to delegate to the Transport layer the choice of the protocol to be used; let us recall that this choice is currently let to the application developer. In [2], the authors assert that the main cause explaining the lack of new Transport protocols deployment and adoption comes from architectural limitations of the Transport layer, hence their proposal for a new architecture. Although this approach is promising, it requires rewriting existing applications and as we previously mentioned, this can slow down its adoption.

\section{Design And Implementation Of The HOOKER FRAMEWORK}

The purpose of this section is to present the internal structure of the Hooker framework and its interactions both with network stack and the applications. Prior to this presentation, it is worth to recall that the Hooker, in its current design, is aimed at demonstrating the concept and hence at opening the way to future performance optimization works.

The Hooker's goals are (1) to redirect TCP connections to another protocol in a transparent manner for the application, and (2) to dynamically deploy the required Transport protocol if it does not already exist in the OS. To do that, the Hooker acts in a different manner on outcoming packets and incoming packets as depicted in Fig. 4, i.e. it places at different levels the hook points and uses several types of eBPF programs associated with those hooks.

On a host running the Linux operating system, the Hooker is attached to root cgroupv2 [14]; hence, by leveraging hierarchical model of cgroups, it can process every ingress and egress packets of all processes running on the host. When the Hooker's eBPF programs are loaded, a map of type SOCKMAP is created. The map key illustrated in code listing 1 is used by the Hooker msg_redirector component to identify the exact socket towards which the packet data must be forwarded to. Each time a connection is established or closed, the map is updated by the Hooker $\mathrm{msg}$ _redirector running SOCK_OPS program attached to cgroupv2.

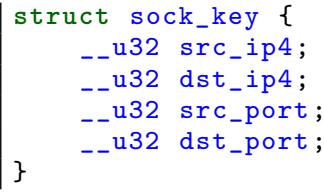

Code listing 1: The structure of the sockmap key

The SK_MSG program running by the Hooker msg_redirector is executed each time an application process sends a message by invoking sendmsg() on a TCP socket. When the Hooker userspace invokes recvmsg(), it receives only the payload without Transport layer address information. By consequence, in order to allow the Hooker userspace to deliver data to the right process when it receives a response from remote pair host, before delivering message to the redirection socket, the Hooker msg_redirector rewrites it by adding one field in the header thanks to buffer extension permitted by the helper function bpf_msg_push_data( ). Finally, to deliver the message either to the redirection socket or to the TCP socket, the Hooker msg_redirector leverages bpf_msg_redirect_map() helper function.

The redirection socket is created and maintained by the Hooker userspace which will use the recv () operation to get the redirected data packet and to send it on the right Transport protocol socket chosen between UDP and UDPLite. In this first prototype, UDP-Lite is dynamically deployed at the same time as the Hooker's eBPF programs. The right moment and the right protocol to use and deploy

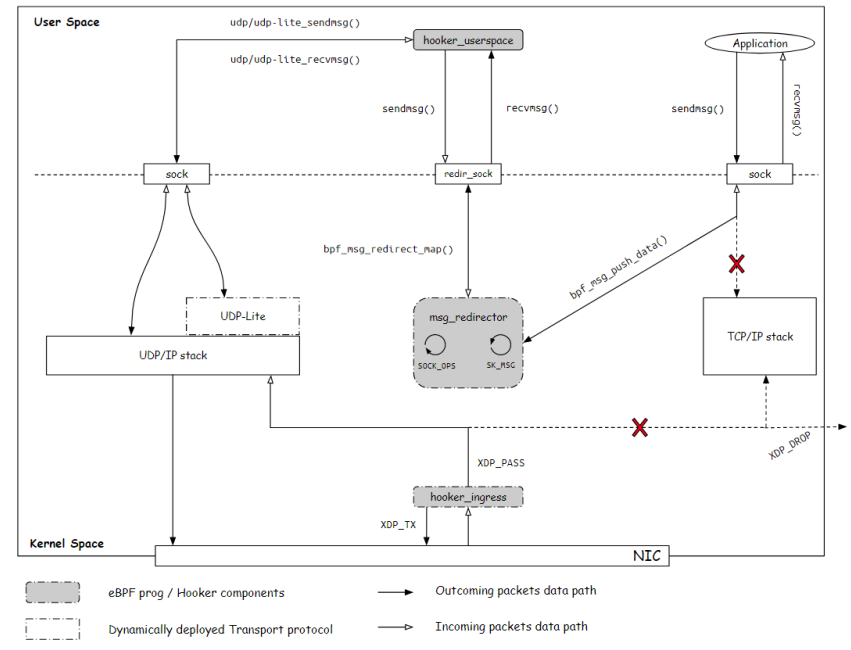

Fig. 4. Hooker Framework Design Overview 
are let to future implementations. The UDP-Lite is chosen because it is a lightweight Transport protocol. Indeed, as stated in section II, to ensure that any eBPF program will terminate within quicktime, the maximum size of each program authorized by the eBPF Verifier is $4096 \mathrm{BPF}$ instructions. However, we can overcome this limitation by partitioning the Transport protocol into small functions and using tail calls feature to chain them as shown in Fig. 1.

For incoming packets, two main hook points can be used, $X D P$ and tc. tc hook has the advantage to be attachable on incoming datapath as well as outcoming datapath; however, it is triggered only after expensive allocation of sk_buff structure by the kernel networking stack. Even if the XDP hook avoids the overheads introduced by allocation of sk_buff structure, currently, it cannot be triggered on outcoming packet data. This is not crippling because outcoming data are hooked from socket layer, hence we use XDP driver hook in this first prototype to leverage it performance and to offset at the best the overheads generate in socket layer by Hooker redirection operations.

Every time, as soon as the host NIC driver receives a packet data, thanks to XDP driver hook, the Hooker egress intercepts the packet data and processes it with the associated eBPF program of type BPF_PROG_TYPE_XDP. The Hooker egress can drop the packet data (XDP_DROP), redirect it to the same or another network interface (XDP_TX, XDP_REDIRECT) or, as currently done in this prototype, pass it to the right network stack (XDP_PASS) for further processing.

\section{Preliminary Tests and Results}

The proposed Hooker prototype is evaluated through experimentations based on data streaming of three files of different types (text, image, and video) having different size, $3 \mathrm{~KB}, 510 \mathrm{~KB}$, and $26 \mathrm{MB}$ respectively. The main goal of these tests is to demonstrate the feasibility of the proposed approach. We measure the cost (delay and overheads) of the redirection and the dynamic deployment of Hooker's programs and the UDP-Lite.

The testbed illustrated in Fig. 5 is composed of two Linux virtual machines (VMs), one acting as the data streaming server and the other one running the client

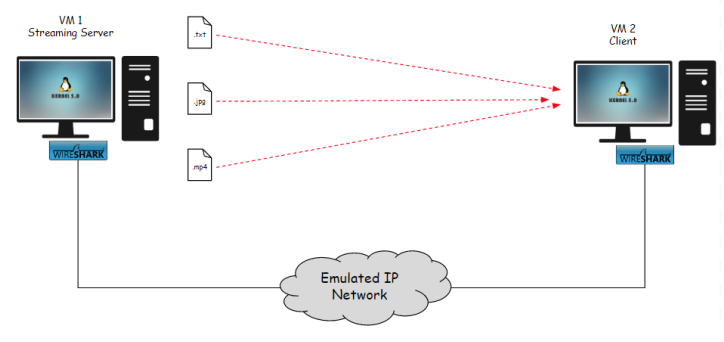

Fig. 5. Experimentations testbed
TABLE I. TESTRED NETWORK AND HOSTS CONFGGRATIONS

\begin{tabular}{|c|c|c|c|}
\hline \multicolumn{4}{|c|}{ VMs specifications } \\
\hline \multicolumn{2}{|c|}{ OS kernel } & CPU & RAM \\
\hline \multicolumn{2}{|c|}{ Linux 5.0} & Intel 2x3.6GHz & 6.3GB \\
\hline \multicolumn{3}{|c|}{ Emulated Network } \\
\hline delay & loss rate & error rate \\
\hline $25 \mathrm{~ms}$ & -- & -- \\
\hline
\end{tabular}

application. The running Linux version for both VMs is 5.0. Each VM is provided with Intel Core i7-7700 CPU and $6.3 \mathrm{~GB}$ of RAM. To make (1) UDP and UDP-Lite as reliable as TCP, and (2) TCP as fast as UDP and UDPLite, we emulated an IP network tuned to zero rate of loss and error. The RTT average is set to $50 \mathrm{~ms}$. See Table 1 for the specifications of VMs and the emulated network.

TABLE II.
\begin{tabular}{|c|c|c|c|}
\hline eBPF programs & Compilation & Loading & Redirection ops \\
\hline Hooker's progs & $6 \mathrm{~s}$ & $20 \mathrm{~ms}$ & $400 \mathrm{~ns}$ \\
\hline UDP-Lite & $200 \mathrm{~ms}$ & $7 \mathrm{~ms}$ & -- \\
\hline
\end{tabular}

For each file, we consider the identical scenario consisting in two steps: the first streaming is performed without running the Hooker and the second streaming is realized with the Hooker running at both sides of the connection (client and server). For each step, we check that the client correctly received the streamed file and with Wireshark analyzer [16], we validate the redirection by checking the Transport protocol used during streaming.

The performed performance evaluation is depicted in Fig. 6. It shows the impact of the Hooker on the transmission latency. Furthermore, we measure the additional overheads introduced by the Hooker framework (see Table 2). The Hooker cost is explained by (1) the redirection operations (multiple memory copies, etc.) and (2) the deployment of eBPF programs operations (compilation and loading). On average, the Hooker introduces $55 \mathrm{~ms}$ additional latency, which is not surprising considering the design and implementation choices made in this first prototype.

\section{Conclusion and Future Research DIRECTIONS}

We propose to use transparent redirection and dynamic deployment to tackle Internet Transport layer innovation issues. We build a first prototype based on eBPF to perform this approach. This prototype, called Hooker, aims to demonstrate the feasibility of our idea and successfully redirect TCP connections to OS native UDP and to UDPLite. The UDP-Lite protocol is dynamically deployed by the Hooker framework. 


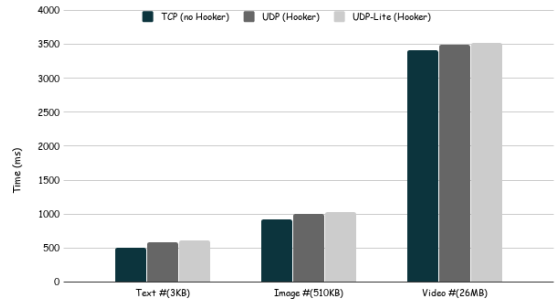

Fig. 6. Latency of the data streaming with and without Hooker

The proposed approach may accelerate the deployment and the adoption of new Transport protocols in the Internet. Furthermore, it can introduce innovation at the Transport layer by allowing the Transport protocol adaptation to the network context and to the application requirements. However, as we show it, the first prototype of the Hooker framework presented in this paper generates additional overheads leading to poverty of provided performances.

One way to optimize the performances is to rethink the architecture of the Hooker, especially its Hooker userspace component which acts currently as "application" proxy. This component may be back to the kernel to act like kernel proxy and so, allow avoiding multiple userspace - kernel space switching contexts. Furthermore, apart from design optimization, we have to rethink the way the eBPFs programs interact with the kernel, to say new helper functions and maps have to be released. For example, here instead of use SOCKMAP to perform the redirection, we can use a shared DATAMAP where the Hooker userspace may directly come to retrieve or to deliver a data and avoid expensive management of multiples buffers socket. This implies the introduction of permanent patches to the Linux kernel.

By showing (1) the benefits of our approach in terms of effective deployment and adoption of Transport protocols and (2) its feasibility, we believe that it will open the way to further optimization research to fully leverage transparent and dynamic deployment of Transport protocols benefits.

\section{REFERENCES}

[1] D. Murray, T. Koziniec, S. Zander, M. Dixon, P. Koutsakis, "An analysis of changing enterprise network traffic characteristics", IEEE Asia-Pacific Conference on Communication (APCC), 2014.

[2] M. Oulmahdi, C. Chassot, N. V. Wambeke, "Transport protocols: limitations, evolution obstacles and solutions for an actual deployment in the Internet", International Journal of Parallel, Emergent and Distributed Systems, Taylor, 2015.

[3] E. Dubois, J. Fasson, C. Donny, E. Chaput, "Enhancing TCP based communications in mobile satellite scenarios: TCP PEPs issues and solutions", Proc. of the 5th ASMS and 11th SPSC, September 2010.

[4] E.-F. Bonfoh, S. Medjiah, C. Chassot, J. Aguilar, "Towards the Virtualization of Transport-level Functions and Protocols", 7th IEEE International Conference on Smart Communications in Network Technologies (SACONET'18), Oct 2018.
[5] M. Handley, "Why the Internet only just works", BT Technology Journal, Vol. 24, No 3, July 2006.

[6] S. McCanne, and V. Jacobson, "The BSD Packet Filter: A New Architecture for User-level Packet Capture", USENIX winter, Vol. 93, 1993.

[7] M. Fleming, "A thorough introduction to eBPF", https://lwn.net/Articles/740157/, accessed 2019-05-08.

[8] T. Høiland-Jørgensen and al., "The eXpress Data Path: Fast Programmable Packet Processing in the Operating System Kernel", 14th ACM International Conference on emerging Networking EXperiments and Technologies (CoNEXT '18), Dec 2018.

[9] "Linux man page", http://man7.org/linux/manpages $/ \mathrm{man} 8 / \mathrm{tc} .8 . \mathrm{html} /$, accessed 2019-05-08.

[10] M. Welzl, F. Niederbacher, S. Gjessing, "Beneficial Transparent Deployment of SCTP: the Missing Pieces", IEEE Global Communications Conference (GlobeCom), December 2011.

[11] R. W. Bickhart and al., "Transparent TCP-to-SCTP translation Shim layer", Unpublished master's thesis, Delaware University, 2005.

[12] N. Khademi et al., "NEAT: A Platform- and Protocol-Independent Internet Transport API", IEEE Com. Magazine, June 2017.

[13] “Transport Services (TAPS)" https://datatracker.ietf.org/wg/taps/about/, accessed 2018-08-05.

[14] "Linux man page", http://man7.org/linux/manpages/man7/cgroups.7.html, accessed 2019-05-08.

[15] BPF and XDP Reference Guide, https://docs.cilium.io/en/v1.5/bpf/\#, accessed 2019-05-09.

[16] Wireshark, https://www.wireshark.org/, accessed 2019-05-08. 